\title{
Modificación de los reglamentos de la Corte Interamericana de Derechos Humanos y de la Comisión Interamericana de Derechos Humanos al procedimiento de peticiones individuales ante la Corte
}

Este comentario, junto a los documentos que analiza, está disponible en www.anuariocdh.uchile.cl

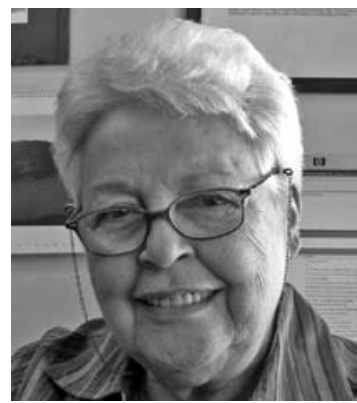

\section{Cecilia Medina Quiroga}

Directora del Centro de Derechos Humanos. Abogada, Licenciada en Ciencias Jurídicas y Sociales por la Universidad de Chile, doctorada en Derecho en la Universidad de Utrecht, Holanda. Es profesora de Derecho Internacional de los Derechos Humanos de la Facultad de Derecho de la Universidad de Chile. Durante ocho años fue miembro del Comité de Derechos Humanos de las Naciones Unidas y presidenta del mismo en los años 1999 y 2000. Fue jueza de la Corte Interamericana de Derechos Humanos desde el año 2002 al 2009.

cmedina@derecho.uchile.cl

\section{RESUMEN}

El artículo revisa los motivos y problemas que subyacen a la modificación de los reglamentos de la Corte Interamericana de Derechos Humanos y de la Comisión Interamericana de Derechos Humanos al procedimiento de peticiones individuales ante la Corte. Asimismo describe las principales modificaciones y novedades introducidas a ambos reglamentos, contextualizándolos con los desafíos que debe enfrentar el sistema interamericano de protección de los derechos humanos.

\section{Introducción}

El sistema interamericano de promoción y protección de los derechos humanos emergió en el continente, de manera poco ortodoxa, con el establecimiento de una Comisión Interamericana de Derechos Humanos (en adelante $\mathrm{CIDH}_{\text {o Comisión) }}{ }^{1}$ antes de existir un tratado de derechos humanos. Más tarde, el sistema fue institucionalizado formalmente por medio de la adopción de un tratado cuando entró en vigor de la Convención Americana (en adelante la Convención o la $\mathrm{CADH})^{2}$ que incorporó a la Comisión y creó la Corte Interamericana de Derechos Humanos (en adelante Corte o Corte IDH). Esto estableció las bases del sistema de protección, que ha ido evolucionando con el paso de los años y con los cambios políticos de la región. Las enmiendas introducidas el año 2009 a los Reglamentos de la Corte y de la Comisión se refirieron a muchos aspectos interesantes de la relación entre ambos órganos y de la manera cómo cada uno ejerce sus funciones. Este artículo, sin embargo, se enfocará exclusivamente al análisis de los cambios que se han producido en el campo de las peticiones individuales como consecuencia de estas enmiendas reglamentarias.

\footnotetext{
1 La Comisión Interamericana de Derechos Humanos fue establecida en 1959 por Resolución VIII de la Quinta Reunión de Consulta de Ministros de Relaciones Exteriores realizada en Santiago, Chile.

2 La Convención Americana sobre Derechos Humanos se adoptó el 22 de noviembre de 1969, durante la Conferencia Especializada de Derechos Humanos, realizada en San José, Costa Rica. Entró en vigor el 18 de julio de 1978.
} 


\section{Relaciones entre la CIDH y la Corte}

Las relaciones entre la Comisión y la Corte se dan solamente en el marco de las peticiones individuales, objeto de este trabajo, y de la jurisdicción no contenciosa de la Corte, que puede ser puesta en movimiento por la Comisión en su calidad de órgano de la OEA ${ }^{3}$.

Para que un caso Ilegue a la Corte es necesario que se hayan completado todas las etapas del procedimiento ante la Comisión ${ }^{4}$. Esto implica la presentación de una denuncia; la posibilidad de que el Estado contra el cual ella se presenta conteste; un período de prueba; la posibilidad de que el Estado y presunta víctima se pongan de acuerdo en un arreglo amistoso; y la adopción de un primer informe por la Comisión y su transmisión al Estado, haciéndole saber, si considera que ha habido una violación, cuál es ella, y haciendo recomendaciones para que sean cumplidas dentro de un plazo 5 .

La CADH no asigna ningún rol explícito a la presunta víctima en el procedimiento individual ante la Corte: no le da locus standi para poner en movimiento la jurisdicción de la Corte, del cual gozan sólo los Estados y la Comisión ${ }^{6}$ y establece en el artículo 57 que esta última debe comparecer en todos los casos que se lleven ante la primera. En términos teóricos, sería posible comparecer ante la Corte en diversas calidades, que en el caso de las peticiones individuales podría ser: a) demandante; b) representante de la víctima, es decir, mandataria que recibe instrucciones de la presunta víctima o c) órgano del sistema.

Desde su primer Reglamento, en $1980^{7}$, la Corte escogió asignar a la Comisión el rol de demandante ${ }^{8}$.

El Reglamento de 1980 de la Comisión permitía enviar como delegado en un caso ante la Corte a cualquier persona que ella designara ${ }^{9}$. Para paliar en algo la nula participación de la presunta víctima, en 1991 se enmendó el Reglamentos de la Corte permitiéndose específicamente incluir entre los delegados de la Comisión a uno o más de los representantes de las presuntas víctimas, lo que les permitió tener un comienzo de participación ante la Corte, aunque siempre dependiente de la Comisión ${ }^{10}$.

Un significativo avance se dio en 2003 cuando la Corte enmendó su Reglamento y dispuso en el artículo 23 un modo de participación de las presuntas víctimas en el proceso ${ }^{11}$. La disposición permitía a las presuntas víctimas someter a la Corte argumentos, mociones y pruebas, aclarándose que de allí en adelante la expresión "partes en el caso" se referiría a la o las presuntas víctimas y a los Estados, reservándose para la Comisión el calificativo de parte procesal. Esta disposición, sin embargo, no alteró la actuación de la Comisión en la práctica ${ }^{12}$.

\footnotetext{
$\mathrm{CADH}$, artículo 35 .

$\mathrm{CADH}$, artículos 48 a 50.

CADH, artículo 50.

CADH, artículo 61.

Corte IDH, Reglamento de 1980. Texto en www.corteidh.or.cr

MEDINA, Cecilia. The bumpy road to human rights. Netherlands Quarterly of Human Rights 26 (4) 2008.

CIDH. Reglamento de 1980, OEA/Ser.L/V/II.49, doc.6 rev. 4, 8 abril 1980.

10 Ver Reglamento de Corte aprobado durante su Período Ordinario de Sesiones XXIII, realizado desde el 9 al 18 de enero, 1991, artículo 22.

11 Corte IDH, Reglamento aprobado por la Corte durante su Período Ordinario XLIX realizado entre noviembre 16al 25, 2000 y enmendado parcialmente durante su Período Ordinario LXI , de noviembre 20 a diciembre 4, 2003, artículo 23.

12 Ibídem, artículo 2.23 .
} 
En esa misma enmienda, la Corte también estableció que, si la demanda de la Comisión no contiene los nombres y dirección de los representantes de la presunta víctima, la Comisión "será la representante procesal de aquéllas como garante del interés público [...] de modo a evitar la indefensión de las mismas"13. Si la presunta víctima tenía representante, éste seguía siendo parte de la delegación de la Comisión ${ }^{14}$. De este modo, la Comisión tomó el curioso papel de ser la demandante propiamente tal, pero, por decisión de la Corte, asumía también la representación de la presunta víctima, pero sin que esta última pudiera decidir dentro del caso actuaciones diferentes a las que deseaba la Comisión.

\section{Los catalizadores de las enmiendas de 2009}

Varios factores se conjugaron para que se impulsara un cambio radical en el mecanismo procesal de las peticiones individuales. El primero que hay que mencionar, es la creciente legitimidad de Comisión y Corte para los que utilizaban el procedimiento y para sus potenciales beneficiarios.

La Comisión tiene diversas funciones y facultades. Examina no sólo peticiones individuales sino que tiene las funciones de promover los derechos humanos, asistir a los Estados cuando éstos lo soliciten, e investigar la situación de derechos humanos en un país o sobre un tema específico. Todas estas funciones se usan en conjunto o separadamente, dependiendo de lo que la Comisión considere más apropiado en cada situación para lograr su objetivo final, que es el de desarrollar el respeto y las garantías de los derechos humanos en el continente.

Las actividades de la Comisión en materia de peticiones individuales y la seriedad con que algunos Estados comenzaron a tratar a la Comisión, permitió, por ejemplo el aumento de las posibilidades de arreglos amistosos que permiten que un caso termine por acuerdo de las partes sin que sea necesario, en principio, Ilegar a la Corte para que el Estado cumpla con sus obligaciones internacionales. Este factor puede haber ayudado a que se incrementara el uso de las peticiones individuales y se ampliara el campo de los asuntos que se Ilevaban ante la Comisión. Otro factor que contribuyó a la utilización del sistema es el surgimiento de organizaciones no gubernamentales que se crearon precisamente con ese fin. Esto a su vez posibilitó desarrollar la crítica al procedimiento para el examen de peticiones individuales.

La Corte, a su vez, apoyó el trabajo de la Comisión y construyó parte de su jurisprudencia sobre él. Hizo y sigue haciendo un trabajo notable al desarrollar el contenido de las reparaciones y de las garantías de no repetición, por nombrar algunos de sus logros. Muy principalmente, ha abierto el camino para que las presuntas víctimas puedan participar cada vez más en los procedimientos contenciosos ante la Corte, permitiéndolas pedir medidas provisionales y presentar solicitudes, argumentos y pruebas. Este último cambió precipitó el debate sobre las posibles fallas del mecanismo.

La principal causa de preocupación para las víctimas provenía de que tenían capacidad para participar en el procedimiento, pero en forma dependiente porque el rol de la Comisión no había cambiado; era ella todavía la demandante, aun cuando el Reglamento de 2003 había caracterizado a la Comisión como parte "solamente procesal". En realidad, el hecho de una doble participación en la representación de los intereses de las víctimas, con la posibilidad de formular peticiones a la Corte y de presentar pruebas, incomodaba a muchos. La inevitable repetición de argumentos, el incremento de presentación de testigos y peritos y de los interrogatorios y contrainterrogatorios

13 Ibídem, artículo 33.3.

14 Ibídem, artículo 22. 
alargaban mucho las audiencias y a menudo las presentaciones e interrogatorios no tenían mayor utilidad $^{15}$.

Los Estados, a su turno, han cambiado su actitud respecto del sistema, desde un casi absoluto ignorar los procedimientos interamericanos a una toma de conciencia de la fuerza y legitimidad que los fallos de la Corte tienen. Ha habido un proceso de reformas constitucionales con relación a los tratados internacionales de derechos humanos y algunos países han dado a la Convención Americana la primera prioridad dentro del ordenamiento jurídico interno. Los Tribunales Supremos y Constitucionales han comenzado a invocar las sentencias de la Corte como una guía para resolver asuntos similares a los tratados en la Corte Interamericana, dando así a la Corte un lugar en el quehacer de los tribunales internos ${ }^{16}$.

Frente a esta evolución, los Estados comenzaron a objetar el hecho de que tanto la Comisión como los representantes de las presuntas víctimas presentaran demandas y pruebas de manera separada. Los Estados consideraban que la igualdad del proceso se había quebrado porque después de la reforma debían litigar contra dos partes. Sólo un Estado intentó lograr un pronunciamiento de la Corte en el sentido de que la participación de la víctima en el procedimiento ante la Corte, en cualquier forma, era violatoria de las normas convencionales. La Corte rechazó esta posición ${ }^{17}$. En cuanto al tema del debido proceso, la Comisión mantuvo que, como las presuntas víctimas son siempre más débiles que el Estado, eso constituía una desigualdad que había que compensar a través de la asistencia directa de la Comisión a la presunta víctima. Además, la Comisión reiteró una posición de muchos años en el sentido de que la verdadera infracción del debido proceso se producía por la facultad que la Corte había dado a los Estados -por una interpretación errónea del artículo 55 de la $\mathrm{CADH}-$, para que estos nombraran a un juez ad hoc para conocer de los casos individuales, lo que les permitía en el hecho tener un juez propio, designado por el Gobierno, para un caso particular en que ese Estado estaba siendo demandado.

En suma, el tema del rol de la Comisión en el procedimiento de comunicaciones individuales ante la Corte comenzó a ser objeto de un intenso debate entre todos los actores del sistema: los Estados, los órganos de supervisión y la sociedad civil, las presuntas víctimas y los Estados, sobre la base de los principios emanados del debido proceso.

Aunque desde el punto de vista de las normas, es correcto sostener que la Comisión es un órgano del sistema, con facultades para supervisar la conducta de los Estados en materia de derechos humanos, y que, por lo tanto, no puede ser la contraparte del Estado en un caso contencioso, no se puede ignorar que las presuntas víctimas son, en general, mucho más débiles que el Estado. Se calcula informalmente que aproximadamente un $40 \%$ de las peticiones que llegan a la Comisión son presentadas por las víctimas mismas, sin representación legal. Desde el punto de vista de la eficacia del sistema, tampoco puede dejar de considerarse que la Comisión tiene un exceso de peticiones en relación con sus recursos humanos y financieros y que los casos que deben ser tramitados por la Comisión sin ayuda de un representante de la presunta víctima, pueden demorar mucho más en avanzar. El envío de un caso a la Corte irroga también gastos que, para los estándares económicos de las víctimas, son inalcanzables. Si la Comisión es la demandante, ella absorbe todos los costos económicos; si deja de serlo, es posible sostener que ya no podría emplear dineros de la Comisión para hacerlo.

\footnotetext{
15 Ibídem, artículos. 35.1 y 3.81.

16 Para información sobre el punto ver NASH, Claudio. La concepción de derechos fundamentales en Latinoamérica. Tendencias jurisprudenciales. México. Editorial Fontamara, 2010.

17 Corte IDH, Caso del Pueblo Saramaka c. Suriname. Sentencia de 28 de noviembre de 2007. Serie C No. 172 , párr. 25.
} 


\section{El proceso de enmienda de los Reglamentos}

El procedimiento que se siguió para reformar los Reglamentos de Corte y Comisión muestra cuánto han avanzado estos dos órganos en sus relaciones y su aguda percepción de que es necesario trabajar conjuntamente como partes de un sistema, tomando también en consideración lo que requieren los Estados y las expectativas de la sociedad civil.

Comisión y Corte trabajaron juntas para acordar los términos de las enmiendas, y Estados y sociedad civil fueron consultados. También se intercambiaron borradores de los Reglamentos respectivos entre Corte y Comisión y se abrió un procedimiento de consulta con los Estados y la sociedad civil.

Una vez recibidos todos los comentarios, cada órgano los procesó. La Corte convocó, además, a una audiencia para escuchar a los Estados y a las organizaciones no gubernamentales que los habían presentado y se produjo un interesante debate. Después de todo esto, cada órgano discutió la versión final y se publicaron los dos reglamentos ${ }^{18}$.

Estimo que todo este procedimiento fue un modo ejemplar de comportamiento que ayuda a transmitir la idea de que en este campo se requiere la cooperación de todos los actores involucrados ${ }^{19}$.

\section{Las enmiendas}

Como ya se ha dicho las enmiendas se motivaron con el fin de reconocer claramente que la Comisión no sería más la contradictora del Estado, y que las presuntas víctimas tendrían una participación plena e independiente, todo esto teniendo como norte el que el sistema interamericano de promoción y protección de los derechos humanos tiene como objetivo principal desarrollar el respeto y goce de los derechos humanos para todas las personas, sin discriminación, y respetar en los procedimientos los principios del debido proceso.

\section{a. Las enmiendas del Reglamento de la Comisión}

En el artículo 69 del Reglamento de 2006 se disponía que la Comisión debían nombrar a sus delegados ante la Corte con el fin de que ellos representaran a la Comisión; los peticionarios podían ser incluidos, pero se sometían en todo a la Comisión. La enmienda de 2009 eliminó la posibilidad de que el peticionario pudiera ser parte de la delegación, liberando así al peticionario de ese problema ${ }^{20}$.

El nuevo Reglamento cambió también la manera en que el caso se remitía a la Corte. La demanda que presentaba la Comisión hasta antes de las nuevas disposiciones, podía diferir en los argumento de derecho (qué derechos se violaban y por qué hechos se violaban) y podía agregar hechos o pruebas que no se habían discutido o presentado durante el procedimiento ante la Comisión. Esto último era, evidentemente, dudoso en términos procesales. La Comisión parecía partir de la base de que el envío del caso a la Corte le daba a éste un nuevo comienzo, lo que no parecía ajustarse a la norma de admisibilidad del artículo 61 de la CADH. Subsistía, sin embargo, el problema de que, a veces, se producían efectivamente hechos y pruebas nuevas después de la adopción del informe del artículo 50; era posible que, tanto los individuos como el Estado, por

\footnotetext{
18 La Comisión adoptó su reglamento reformado en su $137^{\circ}$ período ordinario de sesiones, celebrado del 28 de octubre al 13 de noviembre de 2009. La Corte lo hizo en su LXXXV Período Ordinario de Sesiones celebrado del 16 al 28 de noviembre de 2009.

19 Ver exposición de motivos en el Reglamento de la Corte.

$20 \mathrm{CIDH}$, Reglamento de 2009 artículo 71.
} 
diversas razones, al ver que el caso sería objeto de una controversia judicial, decidieran emplear a fondo todas sus armas disponibles. En ese caso, la Comisión simplemente usaría estos hechos y pruebas nuevas para su demanda.

El Reglamento de 2009 dispone en su artículo 73 que si la Comisión decide enviar el caso a la Corte, notificará de inmediato al Estado, al peticionario y a la víctima ${ }^{21}$ y transmitirá al peticionariotodos los elementos necesarios para la preparación y presentación de la demanda. Está dicho, pues, explícitamente, que no es la Comisión la que presenta la demanda. El artículo 74.1 de este mismo Reglamento establece que el caso se remite a la Corte enviando copia del informe del artículo 50 y del expediente, más cualquier otro documento que la Comisión considere útil. Debe, además, remitirse información sobre su evaluación del grado de cumplimiento de las observaciones contenidas en el informe, el motivo del envío del caso.

La nueva formulación no permite a la Comisión hacer peticiones a la Corte contra el Estado y su papel principal es, en tanto órgano de la Convención, iniciar el procedimiento ante la Corte y explicar las razones del envío de un caso, es decir, la importancia que le asigna a la existencia de una decisión jurisdiccional sobre el tema del caso para el país, un grupo de individuos o la región.

Una vez sometido el caso, se publicará el informe del artículo 50 y la nota de envío ${ }^{22}$. La Corte podrá pedir el envío de otros documentos, pero será la Comisión la que decida si acepta en forma completa o parcial esta petición.

\section{b. Las enmiendas en el Reglamento de la Corte}

Las enmiendas de este Reglamento también apuntan a un papel diferente para la Comisión, que debe, como señala el artículo 57 de la CADH, comparecer en todos los casos ante la Corte.

Como se ha dicho, la Comisión comparecía ante la Corte, curiosamente, como demandante y como representante de las presuntas víctimas. La nueva regulación trata a la Comisión como un órgano del sistema y despareció su calificativo de "parte procesal". La Comisión tiene, a pesar de todo, un papel significativo en el procedimiento.

El procedimiento ante la Corte consta de una etapa escrita y otra oral. En la etapa escrita, y de la misma manera que el Reglamento de la Comisión, el nuevo Reglamento de la Corte señala que el caso se le somete por la Comisión mediante la presentación del informe del artículo 50. Allí deben contenerse todos los hechos presuntamente violatorios y la identificación de las presuntas víctimas ${ }^{23}$. Teniendo en consideración la enorme dificultad de individualizar a las víctimas de violaciones masivas ${ }^{24}$ y justificándose esta circunstancia, la Corte se reserva la posibilidad de decidir posteriormente la calidad de víctimas de personas no identificadas ${ }^{25}$. Deben informarse además los datos de sus representantes, los motivos que la Comisión tuvo para enviar ese caso a la Corte, sus observaciones a la respuesta del Estado sobre las recomendaciones dadas con el

\footnotetext{
21 Nótese que la Comisión habla de víctima y peticionario. La razón de esto es que en el sistema interamericano cualquiera puede presentar una petición, porque existe una actio popularis. Véase artículo 44 de la CADH.

$22 \mathrm{CIDH}$, Reglamento 2009, artículos 74.2 y 3.

23 Corte IDH, Reglamento 2009, artículo 35.1.

24 Piénsese, por ejemplo, en el caso de la Masacre de Plan de Sánchez, donde se exterminó prácticamente en su totalidad una aldea indígena y se quemaron por el Estado los restos humanos que quedaron. Ver Corte IDH, Caso Masacre Plan de Sánchez, Sentencia de 29 de abril de 2004, Serie C No 105.

25 Corte IDH, Reglamento 2009, artículo 35.2.
} 
informe del artículo 50 y sus pretensiones, incluyendo las reparaciones ${ }^{26}$. Pareciera que la solicitud de pretensiones sólo puede entenderse en el sentido de que las razones que tiene la Comisión para llevar un caso a la Corte puedan hacer necesario que ésta ordene medidas más amplias o diferentes de las que se requieren exclusivamente para la situación particular de cada víctima.

Finalmente, la Comisión debe señalar qué hechos del informe somete a la consideración de la Corte $^{27}$; esto porque las funciones de la Comisión exceden con mucho la competencia de la Corte y es posible, que la Comisión envíe un informe que incluya, por ejemplo, asuntos de derechos que están en la Declaración Americana de Derechos del Hombre, pero no en la Convención.

Una vez que el caso está sometido a la Corte, las presuntas víctimas o sus representantes podrán presentar autónomamente su escrito de solicitudes, argumentos y pruebas y proseguir con absoluta independencia todo el proceso $^{28}$. Seguirán, sin embargo, limitadas por los hechos de la contienda presentados por la Comisión.

En la etapa escrita se debe presentar la prueba. La Comisión envía copia de la totalidad del expediente incluyendo toda comunicación posterior al informe del artículo 50 y las pruebas recibidas, indicando cuáles se recibieron en procedimiento contradictorio. Las presuntas víctimas ofrecen su prueba en su escrito de solicitudes, argumentos y pruebas y el Estado lo hace en su contestación a lo señalado por la Comisión y al escrito de las presuntas víctimas ${ }^{29}$.

La Comisión no puede presentar testigos, sino sólo peritos y esto exclusivamente cuando se afecte de manera relevante el orden público interamericano ${ }^{30}$. Por supuesto, el concepto de orden público interamericano es vago, de modo que deberá ser interpretado en cada caso por la Corte. Por lo menos, estimo que ese concepto se basará especialmente en que el la importancia del asunto trasciende el caso particular.

También se han producido cambios en la etapa oral del juicio. La Comisión iniciará esta parte del procedimiento estableciendo los fundamentos del informe y las razones de la presentación del caso ante la Corte $^{31}$. No podrá participar en el interrogatorio de los testigos y peritos que puedan haber ofrecido el Estado y las presuntas víctimas. Sólo podrá interrogar a sus propios peritos presentados con arreglo a lo dicho anteriormente. Podrá hacerlo respecto de los presentados por las presuntas víctimas o por el Estado, sólo con autorización de la Corte, y "cuando se afecte de manera relevante el orden público interamericano de los derechos humanos y su declaración verse sobre alguna materia contenida en un peritaje ofrecido por la Comisión"32.

Al término de los interrogatorios, Estado y presunta víctima -las partes del juicio- presentarán sus alegatos finales. La Comisión podrá presentar observaciones finales ${ }^{33}$.

El procedimiento sigue con una etapa escrita, en que las partes presentan sus alegatos finales escritos y la Comisión puede nuevamente hacer observaciones finales escritas ${ }^{34}$.

\footnotetext{
26 Ibídem, artículo 35.1.

27 Ibídem, artículo 35.3.

28 Ibídem, artículo 25, regulado posteriormente en el artículo 40.

29 Ibídem, artículos 41.

30 Ibídem, artículo 35.f.

31 Ibídem, artículo 51.1.

32 Ibídem, articulo 52.3.

33 Ibídem, artículo 51.

34 Ibídem, artículo 56.
} 


\section{Materias nuevas en el Reglamento de la Corte}

Jueces ad hoc. Hay un punto que merece la pena de ser destacado porque fue un asunto muy discutido que afecta significativamente la igualdad en el proceso, principio inspirador de los cambios. Me refiero a la designación de jueces ad hoc en casos individuales en que una de las partes es un Estado y la otra es una persona. Una vez que se decidió el nuevo papel de la Comisión en el procedimiento ante la Corte, no podía ignorarse el tratamiento de este tema. En realidad, el asunto se refiere no sólo a los jueces ad hoc, sino que a los jueces permanentes nacionales del Estado que está siendo demandado por incumplimiento de sus obligaciones bajo la Convención Americana.

El artículo 55 de la $\mathrm{CADH}$, que regula esta situación, dispone que:

1. El juez que sea nacional de alguno de los Estados Partes en el caso sometido a la Corte, conservará su derecho a conocer del mismo.

2. Si uno de los jueces llamados a conocer del caso fuere de la nacionalidad de uno de los Estados Partes, otro Estado parte en el caso podrá designar a una persona de su elección para que integre la Corte en calidad de juez ad hoc.

3. Si entre los jueces Ilamados a conocer del caso ninguno fuere de la nacionalidad de los Estados Partes, cada uno de éstos podrá designar un juez ad hoc.

Me parece meridianamente claro que consideraciones elementales de un debido proceso prohíben a alguien ser juez de su propia causa. En términos del texto, también parece obvio que este artículo se refiere a casos en que haya dos Estados partes: el número 1 habla de "alguno de los Estados Partes"; el 2 señala que "si uno de los jueces Ilamados a conocer del caso fuere de la nacionalidad de uno de los Estados Partes, otro Estado parte en el caso podrá..."; el 3, "Si [...] ninguno [de los jueces] fuere de la nacionalidad de los Estados Partes, cada uno de éstos podrá designar un juez ad hoc". Esto no es extraño, puesto que se copió del Reglamento de la Corte Internacional de Justicia, cuya base es que dirime contiendas entre Estados y debe respetar el principio de igualdad entre éstos. Cuando no hay dos Estados, no hay razón ni principio alguno que permita que una de las partes, el Estado, nombre un juez en su causa o que un juez de su nacionalidad participe en la causa (aunque no es representante del Estado en su función normal, no hay duda que esta vinculación de nacionalidad puede despertar dudas sobre su imparcialidad).

La Corte, sin embargo, desde su primer caso invitó al Estado (Honduras en el caso) a nombrar un Juez ad hoc y los jueces nacionales siempre permanecieron como tales cuando una causa de su propio Estado se veía. Las razones de esta decisión sólo pueden ser materia de elucubración, porque en su Reglamento la Corte sólo repite lo que dice la Convención y no aporta nada más.

La objeción a los jueces ad hoc empezó a darse por la Comisión en los casos en que la Corte invitaba a un Estado a nombrarlo. En un caso, las presuntas víctimas hicieron lo mismo ${ }^{35}$. A partir de entonces, la objeción se presentó de manera constante, pero sólo ocasionalmente fue mencionada o rechazada. El 14 de agosto de 2008, Argentina decidió ejercitar su derecho a solicitar una opinión consultiva de la Corte respecto del punto.

La Opinión Consultiva № 20 se adoptó el 29 de septiembre de 2009 y concluyó que los jueces ad hoc no eran permisibles en los casos entre un particular y un Estado. Lo mismo debería aplicarse a los jueces nacionales en los casos entre un particular y el Estado de la nacionalidad del juez.

35 Corte IDH; Caso Hermanos Gómez Paquiyauri, Sentencia de 8 de julio de 2004. Serie C No. 110, párr. 22. Ver, por ejemplo, Apitz Barbera y otros. Sentencia de 5 de agosto de 2008. Serie C No. 182, nota al pie 3. 
La Opinión concretó su contenido en el Reglamento de 2009, corrigiendo una situación que, en mi parecer, constituía una flagrante infracción del debido proceso.

El Defensor Interamericano. Otra materia novedosa es la introducción del concepto de Defensor Interamericano, incorporado para solucionar el problema de dar acceso a las presuntas víctimas a la jurisdicción regional, cuando éstas no están en condiciones económicas de hacerlo por sí mismas. El artículo 32 del Reglamento del 2009 dispone que: "En casos de presuntas víctimas sin representación legal debidamente acreditada, el Tribunal podrá designar un Defensor Interamericano de oficio que las represente durante la tramitación de caso".

Se supone que este Defensor será elegido de un grupo selecto de defensores públicos agrupados en la Asociación Interamericana de Defensores Públicos, asociación con la cual la Corte ha firmado un convenio. Es curioso, y puede que no se permita en todos los Estados, que un funcionario público pueda litigar en contra del Estado en un foro internacional; no obstante, ya está sucediendo ${ }^{36}$. El razonamiento de los Defensores es que el sistema regional de protección de derechos humanos es un recurso que los Estados permiten y son esos mismos Estados los que designan a un Defensor para que defienda a un implicado en un juicio criminal dentro del propio país.

Para financiar los costos del procedimiento se ha establecido también un Fondo de Asistencia Legal. Impulsada por los órganos de supervisión regional y por la sociedad civil, la OEA emitió una resolución en 2008 para establecer este Fondo ${ }^{37}$, que debía ser implementado por un Reglamento de la Asamblea General, lo que se hizo en $2009^{38}$ con la cooperación de Corte y Comisión. Los recursos deberán provenir de contribuciones voluntarias de los estados miembros de la OEA y de los Estados que son Observadores Permanente, más los intereses que provengan del capital. Habrá cuentas separadas para Corte y Comisión. La Comisión dictó el Reglamento para la implementación de su cuenta, que entró en vigor el 1 de marzo de 201139; la Corte adoptó el suyo el 4 de febrero de $2010^{40}$, y éste entró en vigencia el 1 de junio de 2010.

No ha transcurrido el tiempo suficiente para hacer un análisis de las bondades o desventajas del Fondo y para evaluar cuánto se demorará en tener un monto de recursos financieros para que pueda operar.

\section{Los desafíos de los cambios}

No será fácil Ilevar a cabo todos estos cambios sin que haya primero procesos de ajuste. Querría destacar aquí sólo un par de puntos. El primero dice relación con la Comisión, que deberá

36 Ver, por ejemplo, Corte IDH, Caso Fermín Ramírez Vs. Guatemala. Sentencia de 20 de junio de 2005. Serie C. No 126, párr. 4, y Caso Raxcacó Reyes Vs. Guatemala. Sentencia de 15 de septiembre de 2005. Serie C. No 133, párr. 4. Ambos casos fueron llevados al Sistema por el Instituto de la Defensa Pública Penal de Guatemala. El Programa para la Aplicación de Tratados de Derechos Humanos de la Defensoría General de la Nación del Ministerio Público de la Defensa Argentina representa a víctimas en treinta y dos peticiones actualmente en trámite ante la Comisión Interamericana de Derechos Humanos. entre ellas, "Fernández Prieto y Tumbeiro vs. Argentina" (caso 12.315) y "Sara del Pilar lbáñez vs. Argentina" (caso 12.710). Informe Anual de la Defensoría General de la Nación Argentina, 2010, páginas 35 y 36, en: http://www. mpd.gov.ar/uploads/Informe_Anual_2010.pdf

37 i.e. OAS AG/RES/ 2426, 3 Cune 2008 no "Creación del Fondo de Asistencia Legal del Sistema Interamericano de Derechos Humanos".

38 OEA, Consejo Permanente, Resolución CP/RES. 963 de 11 noviembre de 2009 que aprobó el Reglamento para el funcionamiento del Fondo de asistencia legal del sistema interamericano de derechos humanos.

39 Reglamento de la Comisión Interamericana de Derechos Humanos sobre el Fondo de Asistencia Legal del Sistema Interamericano de Derechos Humanos. Texto en www.cidh.org.

40 Ver Corte IDH, Reglamento de la Corte Interamericana de Derechos Humanos sobre el funcionamiento del Fondo de Asistencia Legal de Víctimas, 4 de febrero 2010, http://www.corteidh.or.cr/reglamentov.cfm. Entró en vigencia el 1 de junio de 2010 . 
ajustarse a nuevas modalidades de procesamiento de los casos individuales lo que necesariamente le significará más trabajo sin que hayan aumentado sus recursos materiales y humanos. Esto se traducirá, quizás, en una reformulación de actividades a costa de otras labores que son también de primera importancia para la región.

Las presuntas víctimas y las organizaciones que ahora las representan, deberán tener presente que todas sus pruebas deben ser introducidas en el procedimiento ante la Comisión, ya que el informe del artículo 50 determinará el marco en que se llevará a cabo el litigio y no podrán esperar el foro de la Corte para ello. Los Estados deberán hacer lo mismo.

Es una pregunta todavía sin respuesta clara si todos los Estados permitirán a sus Defensores Públicos representar a un nacional ante la Corte. La defensa de nacionales de otros Estados puede ser menos exitosa por el natural desconocimiento de lo que ha sucedido en el caso y cuáles podrían ser los defectos del procedimiento internos en el ámbito de los recursos domésticos. No es aventurado tampoco pensar que los Estados podrían tener intereses de naturaleza geopolítica para no querer que sus defensores públicos representaran a nacionales de ciertos otros Estados.

No es difícil pensar que sería posible que empiece a haber abogados pro-bono -que no tendrían estos problemas- que quieran defender ante la Corte litigantes que carecen de asistencia jurídica. No estarían cubiertos por esta disposición, pero sin duda podría llegarse a un procedimiento similar que repitiera la idea ${ }^{41}$.

Por otra parte, el Defensor Interamericano está concebido para funcionar frente a la Corte, pero el caso se articula y construye en la Comisión. El trabajo ante la Corte está limitado por los hechos que aparezcan en el informe del artículo 50 y estos dependerán mucho de cómo se lleve el caso ante ese órgano. Es posible que la inexistencia del Defensor ante la Comisión vaya en detrimento de la capacidad del Defensor para guiar el caso como le parezca más adecuado para los intereses de las víctimas.

En todo caso, a pesar de los obstáculos que se presentan, las enmiendas en mi parecer van por el camino adecuado: dar cada vez más representación y poder a las presuntas víctimas, lo que contribuye a terminar con infracciones serias del debido proceso. Una de éstas es la falta de independencia de un órgano de la Convención cuando la Comisión se transforma en contraparte de un Estado al cual previamente, y seguramente en el futuro, podría hacer recomendaciones sobre el mismo o sobre otros temas y que en el caso específico le ha dado recomendaciones y señalado plazos para que las cumpla. Otra es la de que el Estado parte en un juicio tenga la posibilidad de nombrar un juez ad hoc para que participe en un caso que le concierne.

Ciertamente estas enmiendas representan un primer intento para dar solución a los problemas a que ellas se dirigen. A medida que se avance en su aplicación, podrán advertirse sus limitaciones. Es de esperar que cuando llegue ese momento, quienes sean miembros de la Comisión o de la Corte perciban esto oportunamente y realicen los cambios que se requieren con el mismo cuidado y respeto por todos los actores del sistema que se tuvo el año 2009.

41 En mi conocimiento, hay ya convenios de la Corte con la Barra Mexicana Colegio de Abogados y con el Colegio de Abogados de Lima. Acuerdo Marco Cooperación entre la Corte Interamericana de Derechos Humanos y la Barra Mexicana Colegio de Abogados, cláusula 2.4, y Acuerdo Marco Cooperación entre la Corte Interamericana de Derechos Humanos y Colegio de Abogados de Lima, cláusula, 2.4. 